\title{
Demonstration of a compressive-sensing Fourier-transform on-chip spectrometer
}

\author{
Hugh Podmore, ${ }^{1, *}$ Alan Scott, ${ }^{2}$ Pavel Cheben, ${ }^{3}$ Aitor V. Velasco, ${ }^{4}$ Jens H. Schmid, ${ }^{3}$ \\ Martin Vachon, ${ }^{3}$ and Regina LeE ${ }^{1}$ \\ ${ }^{1}$ Department of Physics and Astronomy, York University, 4700 Keele St., Toronto, Ontario M1J 1P3, Canada \\ ${ }^{2}$ Honeywell Aerospace, 303 Terry Fox Dr., Kanata, Ontario K2K 3J1, Canada \\ ${ }^{3}$ National Research Council Canada, 1200 Montreal Rd., Ottawa, Ontario K1A 0R6, Canada \\ ${ }^{4}$ Spanish National Research Council (CSIC), Madrid, Spain \\ *Corresponding author: podmore@yorku.ca
}

We demonstrate compressive-sensing (CS) spectroscopy in a planar-waveguide Fourier-transform spectrometer (FTS) device. The spectrometer is implemented as an array of Mach-Zehnder interferometers (MZIs) integrated on a photonic chip. The signal from a set of MZIs is composed of an undersampled discrete Fourier interferogram, which we invert using $1_{1}$-norm minimization to retrieve a sparse input spectrum. To implement this technique, we use a subwavelength-engineered spatial heterodyne FTS on a chip composed of 32 independent MZIs. We demonstrate the retrieval of three sparse input signals by collecting data from restricted sets ( 8 and 14) of MZIs and applying common CS reconstruction techniques to this data. We show that this retrieval maintains the full resolution and bandwidth of the original device, despite a sampling factor as low as one-fourth of a conventional (non-compressive) design. (c) 2017 Optical Society of America

OCIS codes: (130.0130) Integrated optics; (070.2025) Discrete optical signal processing; (120.6200) Spectrometers and spectroscopic instrumentation; (300.6310) Spectroscopy, heterodyne; (300.6300) Spectroscopy, Fourier transforms; (350.4238) Nanophotonics and photonic crystals.

\footnotetext{
Miniature spectrometers are an invaluable tool in many applications, including environmental and biological sensing, medical diagnostics, geology, security, and planetary science, to name a few $[1,2]$. They are particularly sought after in space instrumentation and planetary exploration where it is desirable to minimize the mass and volume of the instruments without compromising performance. Miniature Raman spectrometers are also important for applications in exomineralogy and exobiology [3]. A particularly promising platform for the implementation of miniature spectrometers is planar-waveguide photonic chip technology [4]. In planar-waveguide spectrometers, light is collected and routed through either a dispersive
}

grating element (DE) [5] or a Fourier-transform interferometer array [6].

Of these two architectures, Fourier-transform spectrometers (FTSS) are preferred for their higher optical throughput compared with dispersive grating devices [4,7]. An FTS can be implemented in planar waveguides as an array of Mach-Zehnder interferometers (MZIs) with linearly increasing optical path delays (OPDs) [7,8], also called a spatial heterodyne Fouriertransform spectrometer (SHFTS). In an SHFTS instrument, each point in the spatial interferogram (corresponding to the output of a particular MZI) is captured independently by a linear detector array, allowing for the acquisition of the entire interferogram in a single capture. However, limits on waveguide minimum bend radius, detector size, and fabrication capabilities place constraints on the number of MZIs that may be integrated on a photonic chip, ultimately limiting the bandwidth and the resolution of an SHFTS device. The SHFTS is based on spatial sampling, unlike a scanning FTS [9], where the interferogram is scanned in the temporal domain and captured by a single detector operating at a high readout rate.

Compressive-sensing (CS) techniques can be advantageously used in SHFTS devices, providing a compelling path toward reducing the number of individual interferometers on a planar-waveguide chip and device footprint reduction. In a CS scheme, the input signal is assumed to be sparse, and this assumption is used to significantly reduce the number of sampling points - or MZIs in SHFTS - to retrieve the input signal. CS schemes have already been applied to free-space DE-type systems, but require coded aperture masks which further reduce the optical throughput with respect to the FTS. Fouriertransform devices circumvent mask requirements since the sensing basis (frequency) and the measurement basis (time) are maximally incoherent, i.e., a sparse signal in the frequency domain produces a non-zero signal level at all points in the time domain $[10,11]$. CS schemes are particularly well suited to SHFTS devices, as the sampling points in the interferogram are collected independently. Reducing the number of samples (individual interferometers) in an SHFTS directly reduces the chip footprint. A discretely sampled interferogram consisting of 
$\mathrm{M}$ data points - corresponding to $\mathrm{M}$ individual MZIs - can be reduced to a subset of $\mathrm{K}<\mathrm{M}$ sampling points with no loss in spectral information. This advantage cannot be realized in a scanning FTS, which necessarily collects the full set of sampling points in the time domain.

In this Letter, we demonstrate for the first time, to the best of our knowledge, the CS spectral retrieval of sparse spectra in a waveguide spatial heterodyne FTS device, implemented as an array of MZIs. Our CS SHFTS concept is particularly promising for miniaturized Raman spectrometers, as Raman spectra exhibit a high degree of sparsity [12].

The planar-waveguide spectrometer used in this Letter is composed of an array of 32 unbalanced MZIs with linearly increasing OPDs, as schematically shown in Fig. 1. The OPDs are produced via inducing a change in the optical propagation constant $\beta$ between the two arms of the MZI by a subwavelength grating (SWG), rather than by introducing a geometrical path difference as in conventional devices. One arm of each MZI consists of a standard Si-wire waveguide, while the other arm is an SWG waveguide [13], i.e., a non-diffractive, linear periodic array of Si segments with a period of less than one-half of the effective wavelength to suppress diffraction effects [13]. The SWG structure acts as an optical metamaterial with decreased confinement of the propagating mode, resulting in a reduced group index that can be adjusted by selecting the periodicity and duty cycle of the grating [14,13].

The MZIs are fabricated on a silicon-on-insulator wafer with a $260 \mathrm{~nm}$ thick Si waveguide layer and a $2 \mu \mathrm{m}$ thick buried oxide (BOX). A $2 \mu \mathrm{m}$ thick SU-8 polymer layer is used as the upper cladding. The Si-wire waveguide nominal width is $450 \mathrm{~nm}$, while the SWG grating waveguide has a width of $300 \mathrm{~nm}$, a grating periodicity of $400 \mathrm{~nm}$, and a $50 \%$ fill factor. This results in a group index of $\mathrm{n}_{\mathrm{SWG}}=1.51$ for the $\mathrm{SWG}$ waveguide and $n_{\text {wire }}=4.38$ for the photonic wire waveguide $[13,14]$. The corresponding difference in the group index between the two MZI arms results in the required phase imbalance, controlled across the array by changing the length of the subwavelength region in the delay arm.

The spatial light distribution at the MZI array output ports forms a discretely sampled inverse Fourier transform of the
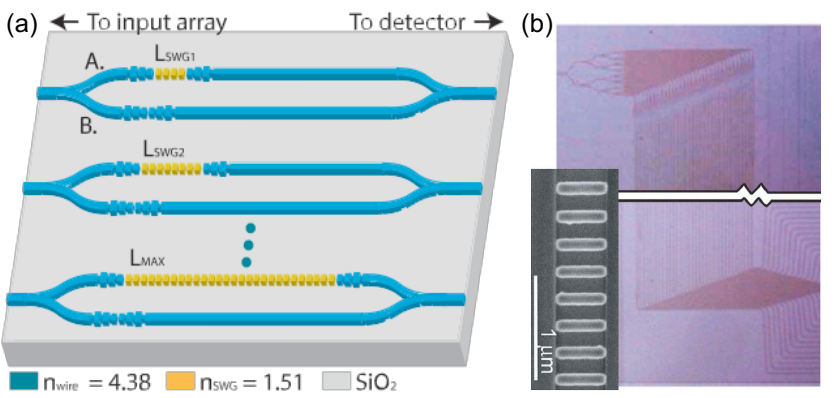

Fig. 1. (a) Light from the spectrometer aperture is directed to an array of interferometers, each consisting of a 50:50 splitter, a delay arm (A), and a reference arm (B). The delay arm in the ith interferometer consists of a pair of subwavelength mode-converters and a SWG region characterized by length $\mathrm{L}_{\mathrm{SWGi}}$. The reference arm consists of a $1.5 \mathrm{~cm}$ long Si-wire waveguide; it also includes a compensatory set of mode-converters connected back-to-back (to balance the loss between the two arms). (b) Fabricated SHFTS chip. Inset: scanning electron microscope image of an SWG waveguide section. input spectra. The MZI number $(\mathrm{M})$ and the OPD physical lengths $\left(\mathrm{L}_{\mathrm{SWGi}}=\mathrm{i} \times \mathrm{L}_{\mathrm{SWG1}}\right)$ are selected in order to set the resolution, $(\delta \lambda)$, and bandwidth, $(\Delta \lambda)$, of the device according to

$$
\begin{gathered}
\delta \lambda=\frac{\lambda_{0}^{2}}{\mathrm{~L}_{\max }\left(\mathrm{n}_{\text {wire }}-\mathrm{n}_{\mathrm{SWG}}\right)} ; \\
\Delta \lambda=\delta \lambda \frac{\mathrm{M}}{2}:
\end{gathered}
$$

where $\lambda_{0}$ is the central wavelength of the device $(1550.5 \mathrm{~nm})$, and $\mathrm{L}_{\max }=\mathrm{M} \times \mathrm{L}_{\mathrm{SWG} 1}$ [8]. In the device under analysis, the OPD lengths increase linearly from $\mathrm{L}_{\mathrm{SWG} 1}=470 \mu \mathrm{m}$, up to a maximum of $\mathrm{L}_{\max }=1.5 \mathrm{~cm}$ for $\mathrm{M}=32$. Experimentally, the SHFTS resolution and the bandwidth were determined as $\delta \lambda=48 \mathrm{pm}$, and $\Delta \lambda=0.78 \mathrm{~nm}$, in good agreement with the theoretical values.

An important advantage of inducing the optical path delays through a change in the mode propagation constant (effective index), rather than a change in the physical path length is that the fringe visibility of the MZIs and, hence, the signal quality, is improved. The visibility factor, $\mathrm{V}=\left(\mathrm{I}_{\max } \mathrm{I}_{\min }\right) /\left(\mathrm{I}_{\max } \mathrm{I}_{\min }\right)$, where $\mathrm{I}_{\max }$ and $\mathrm{I}_{\min }$ are the maximum and minimum MZI output signals, increases as the loss imbalance between the two MZI arms decreases.

In general, the observed fringe maxima and minima in a specific MZI are determined by the propagation loss, $\alpha$, and the physical path lengths of the two arms, z. Since in our FTS with SWG delay lines there is no geometrical path length difference between the two MZI arms, the fringe visibility corresponds to the difference in propagation loss between the SWG and the wire waveguides. In our device, the losses are nearly identical for the SWG and wire waveguides, $\alpha_{\mathrm{SWG}}=$ $-3.0 \mathrm{~dB} / \mathrm{cm}$ and $\alpha_{\text {wire }}=-3.1 \mathrm{~dB} / \mathrm{cm}$, resulting in a measured visibility factor $\mathrm{V}=0.96$.

A high-resolution tunable laser (Agilent 81682A) was used as an input source to characterize the SHFTS chip. The wavelength was scanned in a $5 \mathrm{pm}$ step over a $1 \mathrm{~nm}$ spectral range (encompassing the full bandwidth of the device) for the input power of $1 \mathrm{~mW}$. A polarization controller was used to set the input polarization state to transverse electric (TE), and the light was coupled to the chip via a lensed fiber and on-chip mode transformer [15]. The input power was split on-chip and routed to the individual MZIs using cascaded Y-splitters. The output signal from each output was collimated using a high numerical aperture microscope objective $(\mathrm{NA}=0.4)$, and this collimated beam was captured using a calibrated InGaAs camera (Sensors Unlimited SU320M-1.7RT). Throughout this procedure, the chip temperature was stabilized with a Peltier stage. The product of this characterization (calibration) procedure is an $\mathrm{M} \times \mathrm{N}$ transformation matrix, $\Phi$, in which $M$ is the number of waveguide outputs, and $\mathrm{N}$ is the number of measured wavelength points. The experimental matrix obtained from our SHFTS chip is shown in Fig. 2, demonstrating good fringe visibility and a signal-to-noise ratio for the chosen input power. In this transformation matrix (also called a spectral or calibration map), each row contains the phase and frequency modulation information for a single MZI in the spectrometer. The transformation matrix can be directly used to retrieve an input spectrum via its pseudoinverse [8].

The transformation matrix can be used to implement a CS scheme, wherein an unknown, sparse input spectrum, x, produces an output signal, y, via the sensing scheme: 


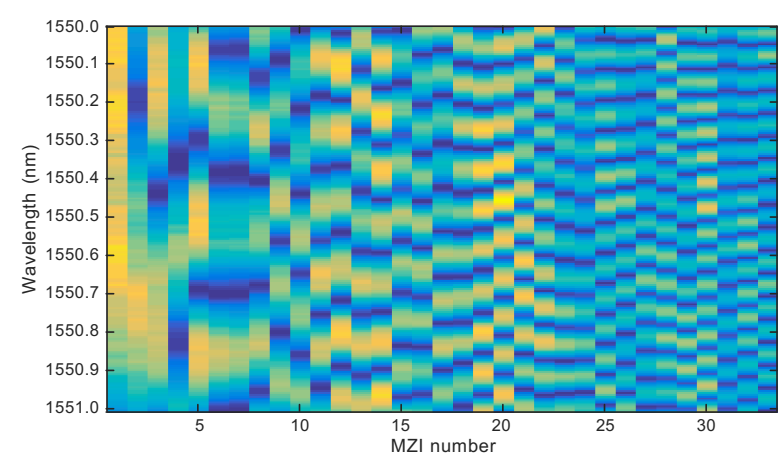

Fig. 2. Measured calibration matrix of the spectrometer chip, showing the wavelength-dependent output power of each of the 32 MZIs as obtained in a high-resolution wavelength scan for a $1 \mathrm{~nm}$ spectral range centered at $1550.5 \mathrm{~nm}$.

$$
\mathrm{y}=\mathrm{Ax}:
$$

In this formulation, $A$ is a $\mathrm{K} \times \mathrm{N}$ matrix with $\mathrm{K}<\mathrm{M}$ that satisfies the restricted isometry property (RIP), i.e., the columns in A are approximately orthogonal. Randomly selected rows of a Fourier-transform matrix are known to satisfy the RIP; hence, a set of K randomly selected MZIs will suffice to define A while satisfying the RIP [16]. Selecting a subset of K rows from the calibration matrix is equivalent to measuring the outputs of a restricted set (K) of MZIs, without considering the remaining $(\mathrm{M}-\mathrm{K}) \mathrm{MZIs}$. Once the output signals of the selected $(\mathrm{K})$ MZIs have been collected, Eq. (3) is solved via $l_{1}$-norm minimization, basis pursuit, to calculate the input spectrum $\mathrm{x}$. In this Letter, the full set of MZIs is included on the chip for the purposes of establishing a baseline comparison, but only a restricted set $(\mathrm{K})$ of MZIs is used to demonstrate the CS scheme spectral retrieval.

First, we consider a monochromatic input spectrum defined by a narrow-band laser source at $\lambda_{0}=1550.5 \mathrm{~nm}$ and $1 \mathrm{~mW}$ input power. Eight MZIs are selected randomly from the interferometer array, their output values are recorded, and the input spectrum is retrieved through $1_{1}$-norm minimization via basispursuit de-noising [10]. This spectrum is shown in Fig. 3

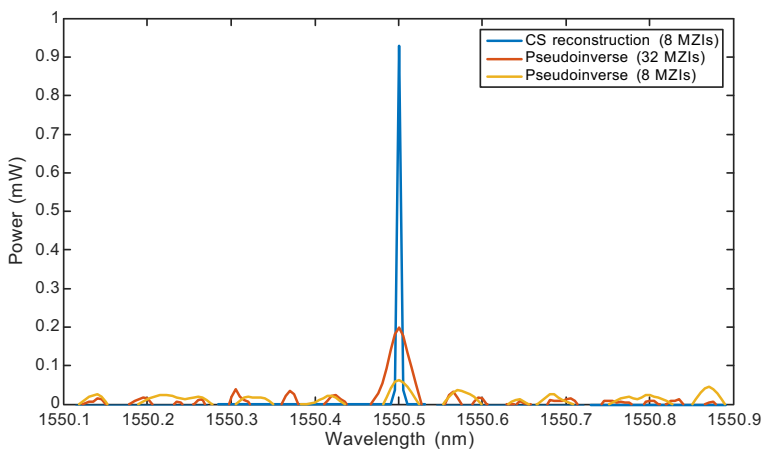

Fig. 3. Spectra from a narrow-band laser source with $1 \mathrm{~mW}$ of input power retrieved using unapodized interferometric measurements on the MZI array. A subset of eight MZIs is randomly selected and their output intensities are used to retrieve the laser spectrum via $1_{1}$-norm minimization (blue). For comparison, the spectrum is also reconstructed via the conventional pseudoinverse method using the full set of 32 MZIs (orange), as well as for the subset of eight MZIs (yellow). beside a retrieval obtained using the pseudoinverse of the calibration map, $\Phi$, as well as the pseudoinverse of A. The retrieval obtained via $1_{1}$-norm minimization lacks the hyperbolic sinusoid ripple associated with an FTS device, which can be observed in the result obtained using the pseudoinverse of the full set of MZIs. The instrument line shape produced by this ripple has a full width at half-maximum (FWHM) of $0.029 \mathrm{pm}$. The lack of ripple in a CS FTS device is a consequence of a priori assumption of a sparse spectrum in the CS schemes. We find that the spectra retrieved via $l_{1}$-norm minimization successfully reconstructs the laser line despite substantial undersampling of the interferogram, with an undersampling factor of $\mathrm{c}=0.25$. By contrast, it is not possible to retrieve a meaningful spectra from the same eight MZIs using the pseudoinverse of the A matrix, as the interferogram is too greatly undersampled for non-CS methods. We note that the total power retrieved in all three methods is preserved ( $1 \mathrm{~mW})$ when integrated within the instrument spectral bandwidth. There is no observable numerical loss in the pseudoinverse retrieval, with the input light intensity being spectrally redistributed due to the line widening, sidelobes, and ripple effects.

In Fig. 4, we present the retrieval of two polychromatic signals (doublets) representing limiting cases of narrowband and broadband signals. The first doublet is spaced at $0.030 \mathrm{~nm}$, closely matching the FWHM of the instrument line shape. The sharp spectral features of the CS retrieval, produced by the a priori assumption of sparsity, allow for clear resolution of the finely spaced doublet; by contrast, the pseudoinverse retrieval is under-resolved with respect to the Rayleigh criterion [17]. A second doublet retrieval with a separation of $0.76 \mathrm{~nm}$, near the bandwidth of the device, is also shown. As in the case of the monochromatic source, it was not possible to retrieve the spectra of either doublet using the pseudoinverse of the matrix $\mathrm{A}$, as the interferogram produced by the 14 MZIs is excessively undersampled.

The results presented in Figs. 3 and 4 demonstrate that the bandwidth and resolution of the CS spectrometer are similar to the conventional non-CS spectrometer, but with a significantly reduced number of interferometers for the CS FTS. The latter requires only 14 MZIs, compared to $32 \mathrm{MZIs}$ for the non-CS

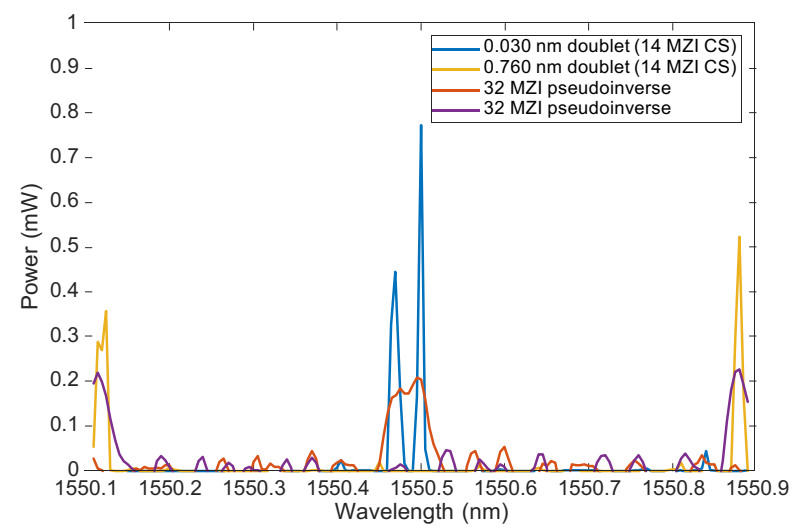

Fig. 4. Spectra from two doublets, with a peak-to-peak separation of 0.030 and $0.76 \mathrm{~nm}$, retrieved using unapodized interferometric measurements of the MZI array. A subset of 14 MZIs is randomly selected, and its output intensities are used to retrieve the spectra via $1_{1}$-norm minimization (blue, yellow). The spectra reconstructed via pseudoinverse methods using the full set of 32 MZIs (orange, purple) are included for comparison. 
FTS in the case of a doublet (undersampling factor $\mathrm{c}=0.44$ ) and only eight MZIs to retrieve a monochromatic source (undersampling factor $c=0.25$ ). The capability of the CS spectrometer to operate with a reduced number of intermediate MZIs also offers a promising path for spectral range expansion at a given resolution, as CS techniques can directly offset the MZI number increase required by Eq. (2). The substantial reduction in the number of sampling interferometers comes with a compromise that the CS spectrometer can only be applied to sparse signals. There are, however, a large number of important naturally occurring sparse spectra. For example, both Raman and laser-induced breakdown spectroscopy (LIBS) techniques produce sparse signals. Strong spectral emission or absorption lines of interest, e.g., to atmospheric science and astronomy may also be considered sparse if a suitable background removal scheme is applied.

In this Letter, we demonstrate that the spatial heterodyne configuration of on-chip FTS devices, consisting of an array of independent interferometers, is uniquely well suited to CS spectroscopy. We use an SHFTS on a photonic chip with an array of MZIs to experimentally demonstrate this technique. SWG-engineered optical delay lines are used in the interferometer arms to minimize the propagation loss imbalance and maximize the fringe visibility of the interferogram. We take advantage of the spatial-heterodyne configuration to independently sample the MZIs and selectively down-sample the spatial interferogram. Using restricted sets of MZIs and $1_{1}$-norm minimization, we retrieve spectra with full resolution and bandwidth, specifically a singlet (with eight MZIs) and a doublet (with 14 MZIs), while a conventional non-CS retrieval requires a substantially larger interferometer array (32 MZIs). These results demonstrate that compressive sensing can be effectively implemented on an FTS chip, allowing a substantial reduction in the number of individual interferometers required for a given resolution and bandwidth. These results open excellent prospects for further miniaturization of integrated FTS devices.

Funding. Natural Sciences and Engineering Research Council of Canada (NSERC); Canadian Space Agency (CSA); Honeywell Aerospace; Ministerio de Economía y Competitividad (MINECO) (FJCI-2014-22836, TEC201571127-C2); Community of Madrid (S2013/MIT-2790); European Association of National Metrology Institutes (H2020-MSCA-RISE-2016: SENSIBLE, JRP-i22 14IND13PhotInd); European Metrology Programme for Innovation and Research (EMPIR).
Acknowledgment. The authors acknowledge Przemek Bock for simulation and mask preparation, and Jean Lapointe for e-beam patterning. A. V. Velasco acknowledges support from the Spanish Ministry of Economy through project TEC2015-71127-C2 and FJCI-2014-22836, the Community of Madrid through project S2013/MIT-2790, EURAMET through project JRP-i22 14IND13-PhotInd and H2020-MSCA-RISE-2016: SENSIBLE, and the EMPIR programme co-financed by the participating states and from the European Union's Horizon 2020 research and innovation program.

\section{REFERENCES}

1. C. P. Bacon, Y. Mattley, and R. DeFrece, Rev. Sci. Instrum. 75, 1 (2004).

2. A. D. Scott, N. Rowlands, and A. Bell, Proc. SPIE 5660, 78 (2004).

3. J. Blacksberg, E. Alerstam, Y. Maruyama, C. J. Cochrane, and G. R. Rossman, Appl. Opt. 55, 739 (2016).

4. M. Florjań czyk, P. Cheben, S. Janz, B. Lamontagne, J. Lapointe, A. Scott, B. Solheim, and D.-X. Xu, Proc. SPIE 7594, 75940R (2010).

5. X. Ma, M. Li, and J. J. He, IEEE Photon. J. 5, 7101307 (2013).

6. K. Okamoto, H. Aoyagi, and K. Takada, Opt. Lett. 35, 2103 (2010).

7. M. Florjań czyk, P. Cheben, S. Janz, A. Scott, B. Solheim, and D.-X. Xu, Opt. Express 15, 18176 (2007).

8. A. V. Velasco, P. Cheben, P. J. Bock, A. Delâge, J. H. Schmid, J. Lapointe, S. Janz, M. L. Calvo, D.-X. Xu, M. Florjań czyk, and M. Vachon, Opt. Lett. 38, 706 (2013).

9. M. Erfan, Y. M. Sabry, M. Sakr, B. Mortada, M. Medhat, and D. Khalil, Appl. Spectrosc. 70, 897 (2016).

10. E. van den Berg and M. P. Friedlander, SIAM J. Sci. Comput. 31, 890 (2008).

11. E. J. Candes and M. B. Wakin, IEEE Signal Process. Mag. 25(2), 21 (2008).

12. O. Katz, J. M. Levitt, and Y. Silberberg, "Frontiers in Optics 2010/ Laser Science XXVI" (2010), paper FTuE3.

13. P. J. Bock, P. Cheben, J. H. Schmid, J. Lapointe, A. Delâge, S. Janz, G. C. Aers, D.-X. Xu, A. Densmore, and T. J. Hall, Opt. Express 18, 20251 (2010).

14. P. J. Bock, P. Cheben, A. V. Velasco, J. H. Schmid, A. Delâge, M Florjańczyk, J. Lapointe, D.-X. Xu, M. Vachon, S. Janz, and M. L. Calvo, Laser Photon. Rev. 7, L67 (2013).

15. P. Cheben, P. J. Bock, J. H. Schmid, J. Lapointe, S. Janz, D.-X. Xu, A. Densmore, A. Delâge, B. Lamontagne, and T. J. Hall, Opt. Lett. 35, 2526 (2010).

16. M. Fornasier, Theoretical Foundations and Numerical Methods for Sparse Recovery (Walter de Gruyter, 2010), Vol. 9.

17. A. W. Jones, J. Bland-Hawthorn, and P. L. Shopbell, Astronomical Data Analysis Software and Systems IV, Astronomical Society of the Pacific Conference Series (1995), Vol. 77, p. 503. 